\title{
Reduction of Major and Minor Hysteresis Loops in a Piezoelectric Actuator
}

\author{
Juan Manuel Cruz-Hernández and Vincent Hayward \\ Centre for Intelligent Machines, \\ 3480 University Street, \\ Montréal, Québec, Canada, H3A 2 A7 \\ manuel@cim.mcgill.ca, hayward@cim.mcgill.ca
}

\begin{abstract}
This paper addresses the compensation of major and minor hysteresis loops using an operator termed a phaser which shifts the phase of a periodic signal by an amount $\phi$. For periodic inputs, hysteresis can be approximated by a phaser with a negative phase shift opening the possibility of cascade compensation. Since in actual hysteresis the phase shift varies according to the size of the input, giving rise to minor loops, the possibility exists to handle the compensation of minor loops by using phasers which vary the phase shift according to the magnitude of the input in an inverse fashion. In this paper, this technique is applied to the compensation of a piezoelectric actuator.
\end{abstract}

\section{Introduction}

In recent years there has been considerably interest in the modeling and control of hysteresis. Specific attention has been given to systems using shape memory alloy (SMA) and ceramic piezoelectric actuators. It is well known that in both type of actuators, the relation of the input-output signals is hysteretic. Hysteresis in those systems is a consequence of the properties of the materials. Short of discovering new materials which are free of hysteresis, correction has to be done by control.

Modeling plays a crucial role in designing controllers and can be approached in two different ways, as is usually the case for the characterization of complex dynamical systems [1]. The model of can be viewed as a collection, or even a continuum, of components for which ideal models can be derived from the principles of physics: the constitutive approach $[2,3,9,11]$. Another approach consists of observing the overall qualitative behavior of the system, and to select some best representative from a class of models that exhibits desired and empirical observed properties: the phenomenological approach ${ }^{1}[10,12,13,18,20]$.

In the literature on the control of hysteresis is customary to obtain a model of the hysteretic plant, then use this model to control it. So is usually the case for the

\footnotetext{
${ }^{1}$ Or the black-box approach
}

control of piezoelectric actuators. In [8], Ge and Jouaneh use a combination of a feed-forward controller (including a nonlinearity) with a feedback loop (PID) to reduce the hysteresis in the actuator represented by the Preisach model. The inverse of this model is used as part of the controller in the form of a look-up table. This technique is also used in $[10,15]$ to compensate the hysteresis in solid state actuators.

Since in general the implementation of a controller is complex, neural networks were also used to compensate for hysteresis, for example hysteresis caused by backlash in mechanical system [16]. Physical analysis of the system nonlinearities and optimal control are used to design the neural network structure.

Adaptive control is also used to control plants with hysteresis behavior. Tao and Kokotović [19] developed control algorithms to reduce the effects of hysteresis-like nonlinearities. The plant consists of a linear part preceded by a hysteresis block representing the actuator. An adaptive hysteresis inverse is cascaded with the plant to cancel the effects of hysteresis so that the remaining part of the controller retains a linear structure. The plant is represented with two nonlinearities, one at the input and one at the output. Model-based nonlinear control methods for magnetostrictive materials are presented in [17]. These are nonlinear optimal control methods which incorporate the hysteresis and nonlinear transducer dynamics.

The present work adopts the phenomenological approach, but no specific model is used to develop a controller, as long as the system can be described by a classical Preisach model. This model is by no means general, but is a convenient tool to analyze the behavior of such a system. It has also been applied successfully in a number of cases $[6,7,14]$, gaining certain degree of generality among several systems.

In a restricted frequency range, it is possible to consider that hysteresis is rate independent and acts as an additive disturbance on the linear dynamics of the system. This is the case for SMA and piezoelectric actuators. Here, a system with hysteresis is seen as a parallel connec- 
Proc. 1998. CDC Conference.

tion of a linear dynamical system with a rate independent hysteresis with memory. In operator form the system can be represented by [4]:

$$
y=\mathbf{L}[u]+\hat{\boldsymbol{\Gamma}}[u]
$$

where $\hat{\boldsymbol{\Gamma}}$ represents the rate independent hysteresis with memory and $\mathbf{L}$ represents the dynamics of the system. This may also be viewed as the two first terms of a Volterra series expansion.

By reduction of hysteresis, we mean a reduction of the area inside the hysteresis loop. If the reduction is complete, an almost univalued relation between the input and output should be obtained. The signals used herein are reciprocating but can have a varying amplitude, this overcomes limitations of controllers implemented in the past [8].

This paper extends the work in $[4,5]$ where the concept of phaser and variable phaser are used. An implementation of the phaser is used to reduce a single hysteresis loop in a nonsaturating piezoelectric actuator. Also an implementation of the variable phaser is used to reduce the hysteresis with saturation found in an SMA actuator at a specific frequency. In both cases the resulting controller relies on the knowledge of the frequency of the input signal. Here, another implementation of the variable phaser is developed and implemented to reduce all the hysteresis loops in a piezoelectric actuator. The implementation of the controller is based on the lead approximations of several phasers, which makes the design easy to understand and implement even if it is a nonlinear controller.

\section{The Piezoelectric Actuator, a System with Hysteresis}

As has been reported in the past $[6,7,10,14]$, hysteresis is a major limitation of piezoelectric actuators. Figure 1 shows the characteristic of a given actuator for a decreasing alternating signal, which increases in frequency as the magnitude decreases.

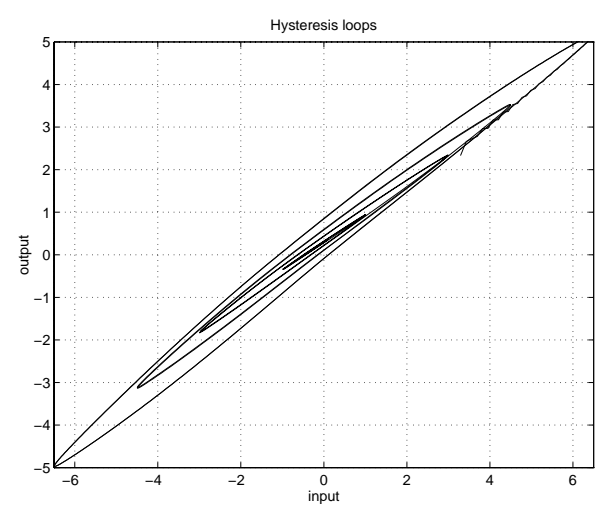

Figure 1: Hysteresis loops of a piezoelectric actuator.

Hysteresis loops are seen as a phase shifts between input and output of the system. Each hysteresis loop in
Figure 1 has a different phase angle, which depends on the magnitude of the input. For larger inputs, the phase angle decreases with the magnitude of the inputs. This can be better seen in Figure 2, where the empirical transfer function estimates are presented for two different input magnitudes. The Bode phase plot shows an almost con-
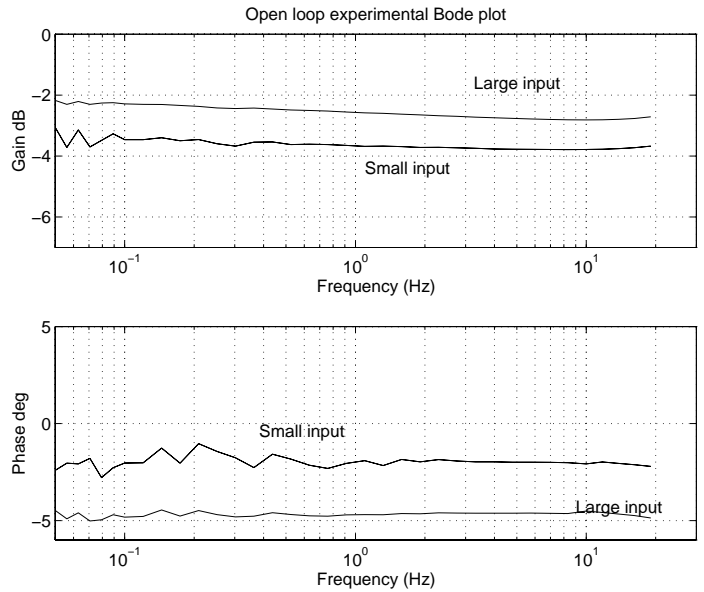

Figure 2: Empirical open loop transfer function. Large input $=6.5$ Volts and Small input $=1$ Volt .

stant angle over the low frequency range for each input magnitude. The bandwidth of this piezoelectric actuator is around $10 \mathrm{~Hz}$.

The objective of the present work is to demonstrate that hysteresis (Figure 1) can be compensated using a variable phaser as a controller.

\section{The Phaser and the Variable Phaser}

In [4], an elementary operator termed a phaser $\left(\mathbf{L}_{p a}\right)$ shifts its periodic input signal by a constant angle $\phi>0$, with a constant magnitude of 1 , independent from the frequency or the magnitude of the input signal.

The frequency domain representation of the phaser is an imaginary number:

$$
\begin{gathered}
\mathbf{L}_{p a}(j \omega)=a+j b \\
\left|\mathbf{L}_{p a}(j \omega)\right|=1 \quad \& \quad \varangle \mathbf{L}_{p a}(j \omega)=\phi \\
a=\frac{\tan \phi}{\sqrt{1+\tan ^{2} \phi}} \quad \& \quad b=\frac{1}{\sqrt{1+\tan ^{2} \phi}}
\end{gathered}
$$

Since it is impossible to realize a causal system that shifts a signal by a constant angle over the whole frequency range, an approximation to the phaser is taken using a series of lead controllers (see Figure 3). This controller shifts the input signal over a finite frequency range, which is chosen to be the bandwidth of the system (usually low frequencies). When the shift angle is small, the phase and magnitude error are quite small. For example, for a shift of 5 degrees and a phase error smaller than a tenth of a degree, the magnitude error is about $1 \mathrm{~dB}$ per decade covered. 
Proc. 1998. CDC Conference.

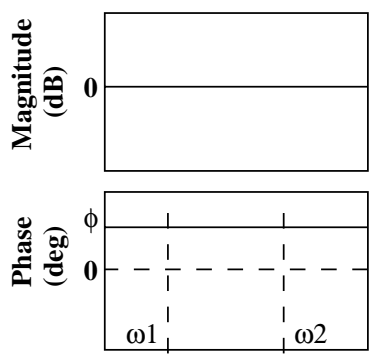

Frequency

a)

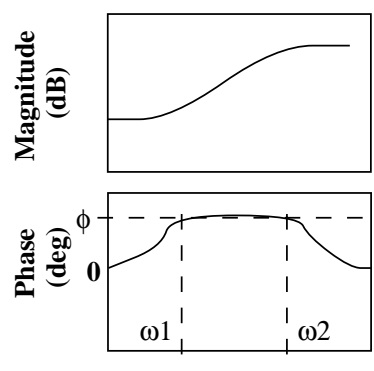

Frequency

b)
Figure 3: Frequency response: a) An ideal phaser. b) An approximation to the phaser over a certain frequency range.

In the design of a practical compensator, $\phi$ is the only parameter to be determined. One way to obtain $\phi$ is to produce an open loop Bode plot of the system (specifically the phase plot in the low frequency range, where the phase is never 0 or a multiple of $90^{\circ}$ ). This method was experimentally applied to a piezoelectric actuator [4], but it has been observed that the phase shift between the input and the output changes as a function of the magnitude of the input. This means that a phaser with a constant angle is not able to reduce all hysteresis loops equally well.

An approximation to the variable phaser was used in [5], to reduce single hysteresis loops with saturation found in smart materials at specific frequencies. In the frequency domain, the ideal variable phaser has the form

$$
\begin{aligned}
& \left.\begin{array}{c}
\mathbf{L}_{p a}(j \omega,|u|)=a(|u|)+j b(|u|) \\
\left|\mathbf{L}_{p a}(j \omega,(|u|))\right|=1 \\
\varangle \mathbf{L}_{p a}(j \omega,|u|)=\phi(|u|)
\end{array}\right\} \\
& a(|u|)=\frac{\tan \phi(|u|)}{\sqrt{1+\tan ^{2} \phi(|u|)}} \\
& b(|u|)=\frac{1}{\sqrt{1+\tan ^{2} \phi(|u|)}},
\end{aligned}
$$

in which the compensation angle varies with the input. The variable phaser can be viewed as an inverse function of the Bode phase plot of Figure 2, while the magnitude is constant for all amplitudes. This can be called phase correction or phase compensation. Since the angle of a variable phaser is varied as a function of the input magnitude, the variable phaser will have different phase angle Bode plots for different input magnitudes and its magnitude plot will be kept constant at $0 \mathrm{~dB}$

\section{Approximation to the Variable Phaser}

Two approximations to the variable phaser are given. The first one depends on the frequency and magnitude of the input signal. The second one is more robust because it can handle input signals of different amplitudes and frequencies between a certain frequency range.

\subsection{PD Implementation}

Equation (2) can be rewritten as [5]:

$$
\mathbf{L}_{p a}(j \omega)=\cos (\phi)+\frac{\sin (\phi)}{\omega} s
$$

where in this case $\cos (\phi)=a, \sin (\phi)=b$ and $s=j \omega$, or in the time domain:

$$
y(t)=\cos (\phi) u(t)+\frac{\sin (\phi)}{\omega} \dot{u}(t)
$$

where $y(t)$ is the output, $\phi$ is the phase lag between the input and the output, $\omega$ is the frequency of the input signal, and $u(t)$ is the input signal. This representation has the form of a PD controller.

If the angle $\phi$ varies with the input magnitude, equation (4) becomes

$$
\mathbf{L}_{p a}(j \omega,|u(t)|)=\cos (\phi(|u(t)|))+\frac{\sin (\phi(|u(t)|))}{\omega} s
$$

where the angle varies as a function of the input magnitude, to yield a variable phaser. This implementation was used in [5]. This controller must be designed for an specific input frequency, and if the input contains more than one frequency, the controller will not compensate well for that signal. Nevertheless, if it is known that the signal has a given frequency, this implementation gives very good results.

\subsection{Lead Implementation}

The phaser can also be implemented by a linear filter of the form

$$
L_{p a}(s)=\frac{s^{n}+a_{n-1} s^{n-1}+\ldots+a_{1} s+a_{0}}{s^{n}+b_{n-1} s^{n-1}+\ldots+b_{1} s+b_{0}}
$$

where the coefficients are chosen such that the phase of the filter is almost constant around the design parameter $\phi$ within an operational range of frequencies $\omega_{1}$ and $\omega_{2}$ (see Figure 3). This filter can be implemented by fixing the values of its $n$ zeros to lie between $\omega_{1}$ and $\omega_{2}$. Then, the $n$ poles are found to achieve a phase angle centered on $\phi$, between $\omega_{1}$ and $\omega_{2}$.

The phase angle $\phi_{v a r}(|u|)$ for the variable phaser depends on the magnitude of the input signal. The operational range is as before, $\omega_{1}$ and $\omega_{2}$. To achieve an approximation to the variable phaser independent of the input frequency, the denominator is made to depend on the magnitude of the input signal.

$L_{p a}(s,|u(t)|)=\frac{s^{n}+a_{n-1} s^{n-1}+\ldots+a_{1} s+a_{0}}{s^{n}+b_{n-1}(|u(t)|) s^{n-1}+\ldots+b_{0}(|u(t)|)}$

The coefficients $b_{i}(|u|)$ are derived from several phasers with different angles $\phi$, corresponding to different magnitudes of the input signal. Their numerators are 
Proc. 1998. CDC Conference.

kept fixed since all phasers are designed for the same operating range, and because the denominators determine the phase angle. For $m$ different input magnitudes, the following filters are designed:

$$
\begin{aligned}
L_{p a_{1}}(s)= & \frac{s^{n}+a_{n-1} s^{n-1}+\ldots+a_{1} s+a_{0}}{s^{n}+b_{n-1}^{1} s^{n-1}+\ldots+b_{0}^{1}} \\
L_{p a_{2}}(s)= & \frac{s^{n}+a_{n-1} s^{n-1}+\ldots+a_{1} s+a_{0}}{s^{n}+b_{n-1}^{2} s^{n-1}+\ldots+b_{0}^{2}} \\
\vdots & \vdots \\
L_{p a_{m}}(s)= & \frac{s^{n}+a_{n-1} s^{n-1}+\ldots+a_{1} s+a_{0}}{s^{n}+b_{n-1}^{m} s^{n-1}+\ldots+b_{0}^{m}}
\end{aligned}
$$

with different phase angles. A function $b_{i}(|u|)$ is fitted using all the values $b_{i}^{j}$ corresponding to each magnitude $j$, for $i=0, \ldots, n-1$ and $j=1, \ldots, m$, where $n$ is the order of the filter and $m$ the number of input magnitudes considered. The variation of $L_{p a}(s,|u(t)|)$ with the size of the input signal means that the phase angle will vary with the size of the input signal, which is done by varying the coefficients of the denominator. The complexity will depend on the type of approximation taken to obtain $b_{i}(|u|)$.

If individual phasers are designed to be stable such that poles and zeros are located in the left hand side of the complex plane, with proper interpolation, the resulting variable phaser will be stable too since the coefficients will never leave the left hand side of the complex plane.

To end this section, the phase loops of a constant and variable phasers are compared in Figure 4. The loop of the variable phaser is deformed whereas the loop of the constant phaser is perfectly elliptic.

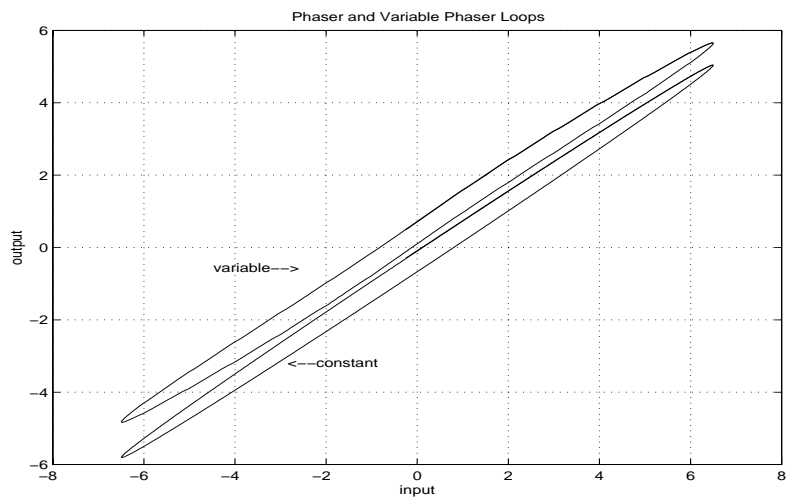

Figure 4: Loops of the phaser and variable phaser.

The small distortion which is noticed on the loop of the variable phaser in Figure 4 is due to the fact that the angle varies continuously with the input whereas it should change only at extrema of the input. In practice, this defect is corrected by the feedback regulation, as will be demonstrated by the experimental results. However, the variable phaser implementation can be further improved by changing the angle at reversals only, that is when the input goes through a local extremum and when this extremum is smaller than the previous one. This additional complication is not necessarily needed in practice, since if its is implemented the output will not be smooth and some jumps will appear at the time the phaser changes its phase angle [5].

\section{Case Study}

A $4 \mathrm{~cm}$ lead zirconate titanate bimorph piezoceramic actuator has been used to implement the variable phaser described above. Its characteristics were presented in Figure 1 using a signal which generates major and minor loops ${ }^{2}$. The signal contains several frequencies so the dynamic behavior of the controller can be tested. They lie in the bandwidth of the system and within the range of action of the controller.

The bandwidth of the actuator is around $10 \mathrm{~Hz}$ so the controller is designed to lie between $\omega_{1}=0.05 \mathrm{~Hz}$. and $\omega_{2}=5 \mathrm{~Hz}$. The order of the lead controllers was chosen to be $n=4$ and the number of input magnitudes $m=3$. The variation of the phase angle $\phi$ used in the implementation of the variable phaser is presented in Figure 5.

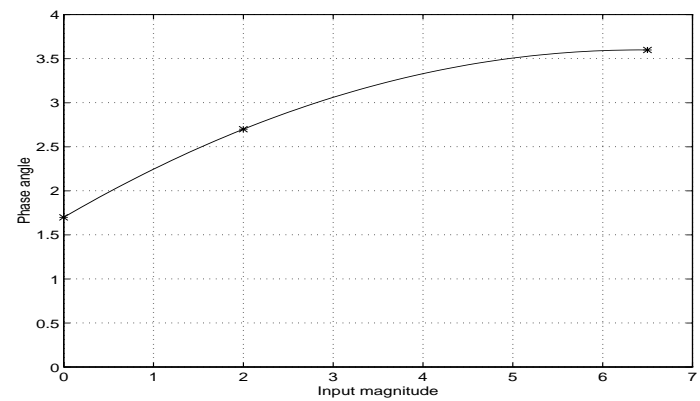

Figure 5: Variation of the phase angle as a function of the input magnitude $-\mathrm{u}(\mathrm{t})$ -

The form of the variable phaser is:

$$
\begin{aligned}
L_{p a}(s,|u(t)|) & =\frac{s^{4}+39.87 s^{3}+277.65 s^{2}+373.28 s+73.05}{s^{4}+b_{3}(|u(t)|) s^{3}+\ldots+b_{0}(|u(t)|)} \\
b_{3}(|u(t)|) & =-0.0456|u(t)|^{2}+0.5964|u(t)|+41.569 \\
b_{2}(|u(t)|) & =-0.5622|u(t)|^{2}+7.4784|u(t)|+298.248 \\
b_{1}(|u(t)|) & =-1.0929|u(t)|^{2}+14.9302|u(t)|+414.089 \\
b_{0}(|u(t)|) & =-0.3143|u(t)|^{2}+4.3630|u(t)|+84.741
\end{aligned}
$$

The implementation of a constant controller with $\phi=3$, is used to compare the performance of the variable phaser. The constant phaser is:

$L_{p a}(s,|u(t)|)=\frac{s^{4}+39.87 s^{3}+277.65 s^{2}+373.28 s+73.05}{s^{4}+42.88 s^{3}+314.89 s^{2}+447.95 s+94.81}$

Note that the numerator in both constant and variable phasers is the same, since the phase will be given by the denominator.

\footnotetext{
${ }^{2}$ The input signal had magnitudes of $6.5,4.5,3.0$ and 1.0 volts, with corresponding frequencies $0.05,0.1,0.5$ and $1 \mathrm{~Hz}$ respectively
} 
Proc. 1998. CDC Conference.

Using the same input as before, Figures 6 , and 7 are obtained after using the constant and variable phasers.

a)

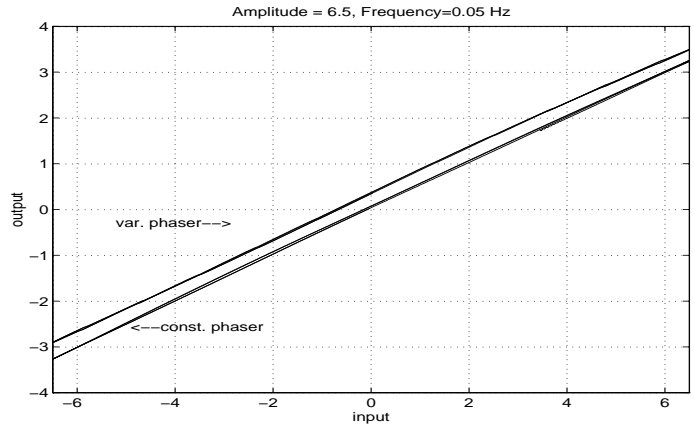

b)

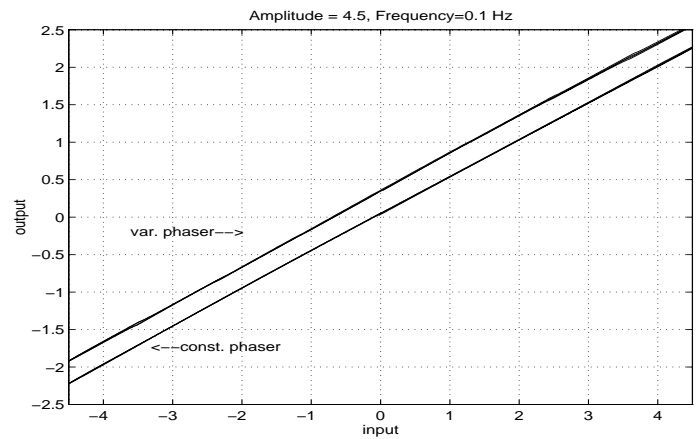

Figure 6: Hysteresis loop after using the constant and variable phaser for a) an amplitude $=6.5 \mathrm{~V}$ and Frequency $=0.05 \mathrm{~Hz}$, and $\mathrm{b}$ ) an amplitude $=4.5 \mathrm{~V}$, Frequency $=0.1 \mathrm{~Hz}$.

a)

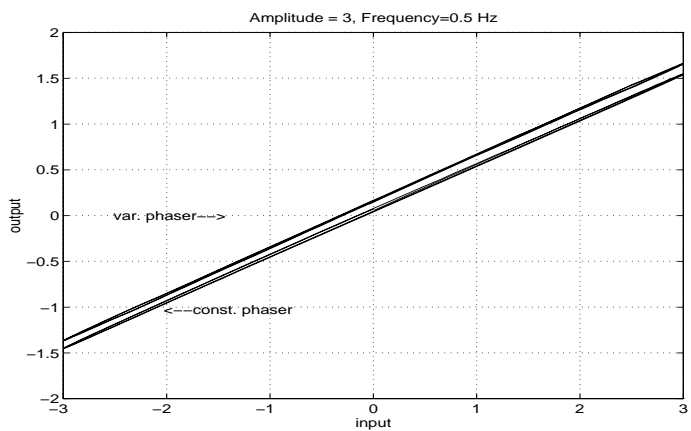

b)

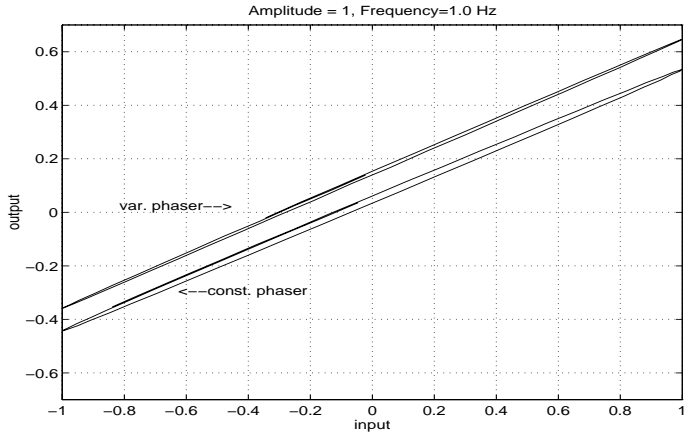

Figure 7: Hysteresis loop after using the constant and variable phaser for a) an amplitude $=3.0 \mathrm{~V}$, Frequency $=0.5 \mathrm{~Hz}$, and b) an amplitude $=1.0 \mathrm{~V}$, Frequency $=1 \mathrm{~Hz}$.

These resulting figures show a better compensation when using a variable phaser than when using a constant phaser. Using an increasing signal, similar results are obtained as the ones obtained with the decreasing signal.

The empirical transfer function Bode plots are presented in Figure 8.
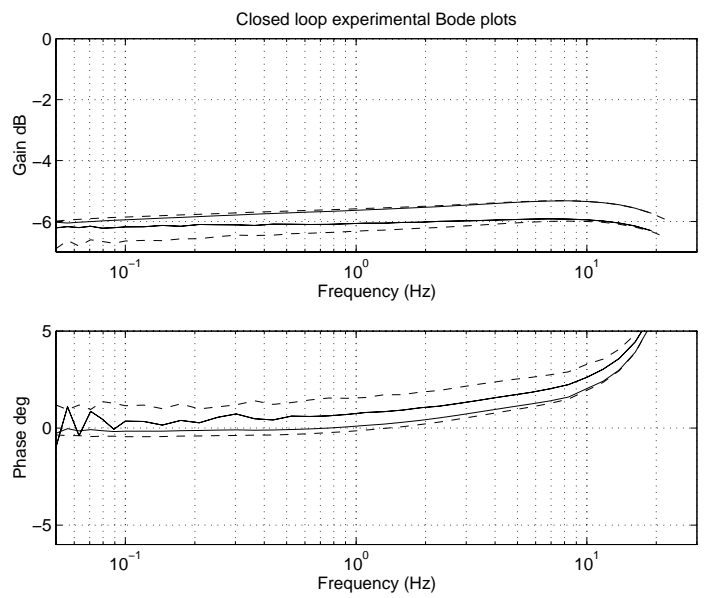

Figure 8: Empirical closed loop transfer function. Dashed line: using a constant phaser; continuous line: using a variable phaser.

The magnitude and phase uncertainties between $\omega_{1}$ and $\omega_{2}$ is smaller when a variable phaser is used than with the constant phaser.

A set of figures showing the performance of both controllers for different input magnitudes and frequencies is presented in Figure 9.

\section{Conclusions}

A control design strategy has been introduced to reduce hysteresis. The approach uses the so-called variable phaser, an operator that shifts its periodic input signal by a phase angle $\phi$ that depends on the magnitude of the input signal. An approximation to the ideal variable phaser was introduced by using the lead controller with a denominator with variable coefficients. A variable phaser was designed to reduce the hysteresis found in piezoelectric actuator. The results are remarkably good, since almost a linear input-output relation was obtained, for any input amplitude and any frequency between the operational range of the controller.

Current work is carried out toward the development of a measure of performance, to quantify and compare different controllers.

\section{Acknowledgements}

This research was funded by an operating grant from NSERC, the National Science and Engineering Council of Canada and by IRIS (Phase II), the Institute for Robotics and Intelligent Systems part of Canada's Centres of Excellence Program (NCE), project "MSA-4". 
Proc. 1998. CDC Conference.
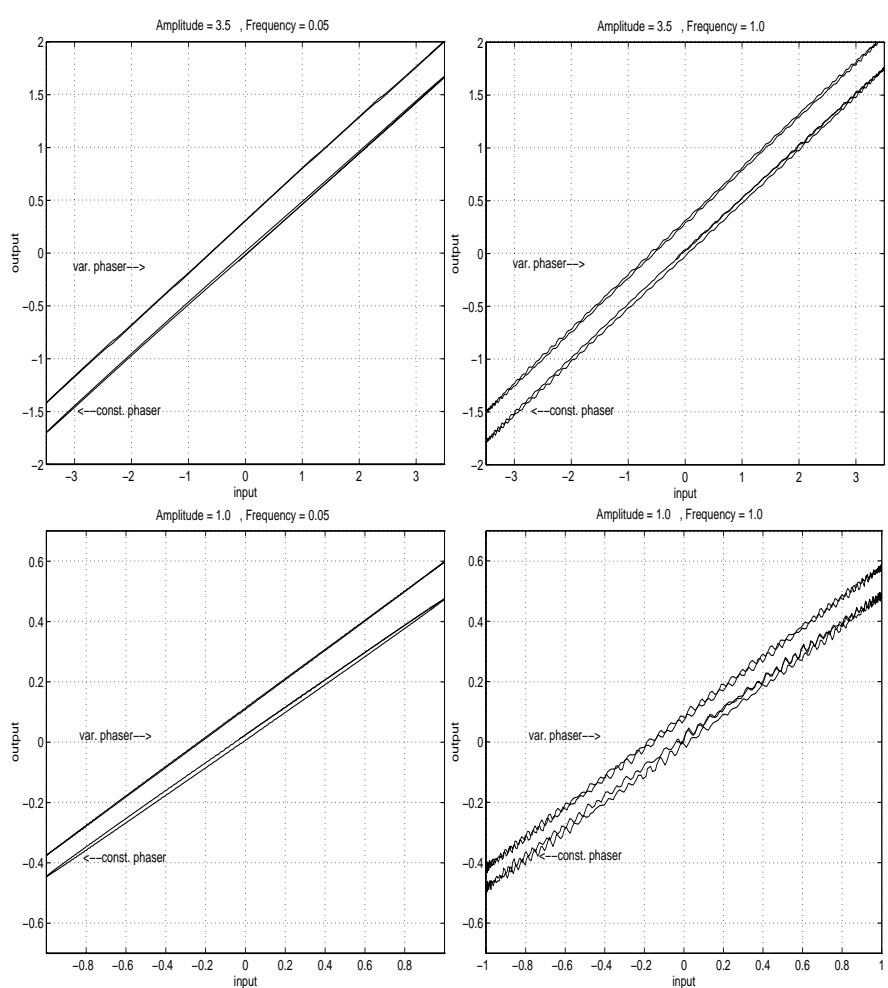

Figure 9: Loops for different input magnitudes and frequencies.

\section{References}

[1] H.T. Banks, A.J. Kurdila, and G. Webb. Identification of hysteresis control influence operators representing smart actuators. part i: Formulation. Technical report, Center for Research in Scientific Computation, North Carolina State University, CRSC-TR9614. October, 1996.

[2] M. Brokate and J. Sprekels. Hysteresis and phase transitions. New York : Springer, 1996.

[3] L.O. Chua and S.C. Bass. A generalized hysteresis model. IEEE Transactions on Circuit Theory, CT19(1):36-48, January 1972.

[4] J.M. Cruz-Hernández and V. Hayward. On the linear compensation of hysteresis. In 36th IEEE Conference on Decision and Control, volume 1, pages 1956-1957, 1997.

[5] J.M. Cruz-Hernández and V. Hayward. An approach to reduction of hysteresis in smart materials. In 1998 IEEE International Conference on Robotics and Automation, pages 1510-1515, 1998.

[6] A.R. Freeman and S.P. Joshi. Numerical modeling of pzt nonlinear electromechanical behavior. SPIE, Smart Structures and Materials, 2715:602-613, 1996.
[7] P. Ge and M. Jouaneh. Modeling hysteresis in piezoceramic actuators. Precision Engineering, 17(3):211221, July 1995 .

[8] P. Ge and M. Jouaneh. Tracking control of a piezoceramic actuator. IEEE Transactions on Control Systems Technology, 4(3):209-216, May 1996.

[9] M. Goldfarb and N. Celanovic. Behavioral implications of piezoelectric stack actuators for control of micromanipulation. In Proceedings of the IEEE International Conference on Robotics and Automation, pages 226-231, 1996.

[10] D. Hughes and T.J. Wen. Preisach modeling and compensation for smart material hysteresis. SPIE, Active Materials and Smart Structures, 2427:50-64, 1995.

[11] M.P. Kennedy and Chua L.O. Hysteresis in electronic circuits: A circuit theorist's perspective. International Journal of Circuit Theory and Applications, 19:471-515, 1991.

[12] M. Krasnosel'skiü and A. Pokrovskiü. Systems with Hysteresis. Berlin; New York: Springer-Verlag, 1989.

[13] I.D. Mayergoyz. Mathematical Models of Hysteresis. Springer-Verlag, 1991.

[14] P. Ortin. Preisach modeling of hysteresis for pseudoelstic cu-zn-al single crystal. Journal of Applied Physics, 71(3):1454-1461, February 1992.

[15] J. Schafer and H. Janocha. Compensation of hysteresis in solid state actuators. Sensors and Actuators A, (Physical), 49:97-102, 1995.

[16] D.R. Seidl, S.L. Lam, J.A. Putman, and R.D. Lorenz. Neural network compensation of gear backlash hysteresis in position-controlled mechanisms. IEEE Transactions on Industry Applications, 31(6):14751482, November/December 1995.

[17] R.C. Smith. A nonlinear physics-based optimal control method for magnetostrictive actuators. Technical report, Center for Research in Scientific Computation, North Carolina State University, CRSC-TR9733. October, 1997.

[18] R.C. Smith. Modeling techniques for magnetostrictive actuators. Technical report, Center for Research in Scientific Computation, North Carolina State University, CRSC-TR97-6. March, 1997.

[19] G. Tao and P.V. Kokotović. Adaptive Control of Systems with Actuator and Sensor Nonlinearities. Wiley, 1996.

[20] A. Visintin. Differential Models of Hysteresis. Springer-Verlag, 1991. 